\title{
Strangeness equilibration in heavy ion collisions
}

\author{
Subrata Pal, C. M. Ko, and Zi-wei Lin \\ Cyclotron Institute and Physics Department, Texas A\&M University, College Station, Texas 77843-3366
}

(Received 15 May 2001; published 19 September 2001)

\begin{abstract}
Using a relativistic transport model for heavy ion collisions at energies that are below the threshold for kaon and antikaon production in nucleon-nucleon collisions, we study how their abundances approach the canonical equilibrium during the collisions. We find that kaons are far from chemical equilibrium at the initial and high density stage, and they approach equilibrium only during the expansion stage of the collisions when their production rate is small and becomes comparable to their annihilation rate. In contrast, antikaons approach chemical equilibrium much earlier but eventually fall out of equilibrium again as a result of their large annihilation cross sections in nuclear matter.
\end{abstract}

DOI: 10.1103/PhysRevC.64.042201

PACS number(s): 25.75.Dw, 24.10.Jv, 24.10.Lx

Recent analyses have shown that most hadrons measured in heavy ion collisions can be described by statistical models based on the grand canonical ensemble for abundant particles [1] and the canonical ensemble for rare particles [2]. On the other hand, studies based on transport model indicate that the chemical equilibration time for kaons and antikaons in the hot dense matter, which is expected to be formed in heavy ion collisions at the AGS and SPS energies, is an order of magnitude longer than the heavy ion collision time [3]. It has thus been suggested [4] that the kaon equilibration time can be significantly shortened if the kaon mass is reduced in dense matter as a result of the large attractive scalar interaction and diminishing repulsive vector interaction due to the assumption of vector decoupling. The disappearance of the repulsive vector interaction is, however, not consistent with the strong repulsive vector potential that is needed to understand the vanishing kaon flow observed in heavy ion collisions at GSI energies [5,6] and the large kaon antiflow observed in heavy ion collisions at AGS energies [7,8]. Also, a recent transport model study has shown that the measured kaon yield in heavy ion collisions at GSI [9] can be explained with kaons interacting with both the scalar and vector potentials [10].

To gain insight into this problem, a kinetic theory for the time evolution of particle production [11] can be applied. It is found that the equilibrium time $\tau_{0}^{\mathrm{C}}$ for rare particles carrying $U(1)$ charge and described by the canonical ensemble due to $U(1)$ charge conservation is much shorter than what is expected from the grand canonical ensemble, i.e.,

$$
\tau_{0}^{\mathrm{C}}=\tau_{0}^{\mathrm{GC}} N_{\mathrm{eq}}^{\mathrm{GC}} \ll \tau_{0}^{\mathrm{GC}},
$$

where $N_{\text {eq }}^{\mathrm{GC}}$ is the average multiplicity of rare particles per event if they were described by the grand canonical ensemble. Also, it is shown that

$$
N_{\text {eq }}^{\mathrm{C}}=\left(N_{\text {eq }}^{\mathrm{GC}}\right)^{2} \ll N_{\text {eq }}^{\mathrm{GC}},
$$

so the equilibrium multiplicity in the canonical ensemble is much lower than that given by the grand canonical ensemble.

The above idea based on the kinetic theory can be quantitatively studied using the transport models, which have been very successful in understanding many aspects of heavy ion collisions [12]. In this Rapid Communication, we shall use the transport model to study kaon and antikaon production in heavy ion collisions at energies that are below the thresholds for their production in nucleon-nucleon interactions, and to understand how kaons and antikaons approach chemical equilibrium in these collisions. In previous studies of subthreshold kaon production using transport models [13$16]$, only the production of kaons has been included while their annihilation via the inverse reactions has been neglected. The neglect of kaon annihilation would not be a bad approximation if kaons are far from chemical equilibrium. However, to understand kaon chemical equilibration in transport models, we need to include kaon annihilation to see how the kaon production and annihilation rates become comparable during the finite heavy ion collision time.

We follow essentially the same model used in Ref. [17], which is based on the transport model relativistic VlasovUehling-Uhlenbeck (RVUU) [18]. In this model, the nuclear potential is taken from the nonlinear Walecka model, so it has both an attractive scalar and a repulsive vector part. The attractive scalar potential allows one to treat consistently the change of nucleon mass in nuclear matter. In dense matter, the nucleon mass is reduced and the energy is in the scalar field. As the system expands, the nucleon regain its mass from the scalar field energy. The model in Ref. [17] includes kaon production from both baryon-baryon and meson-baryon interactions. For kaon production from baryon-baryon interactions, the cross sections are taken from the predictions of the meson-exchange model introduced in Ref. [19]. For kaon production cross sections from meson-baryon interactions, they are obtained from the resonance model of Ref. [20]. The produced kaons together with their partners, mainly hyperons, not only undergo elastic scatterings with baryons but are also affected by mean-field potentials. For kaon and hyperon scattering cross sections, we take the empirical values as in Ref. [17]. The kaon potential is taken from the chiral Lagrangian including both scalar and vector interactions [21] with their strengths determined from experimental observables such as the kaon yield and collective flow in heavy ion collisions [17].

The scattering of kaon with pion, which is mainly elastic through the $K^{*}$ resonance, is neglected in the model. Since pions appear only at later stage of the collision from delta 
decays and their abundance is much smaller than that of nucleons in heavy ion collisions at GSI energies, the effect of kaon-pion scattering has been shown to be unimportant [14]. In particular, it should have negligible effect on the chemical equilibration of kaons to be studied here.

Also, the off-shell effect on baryons due to their scatterings is not considered in this model. Since the imaginary self-energy resulting from scatterings leads to a broad spectral distribution for a baryon, baryons with free-space energies below the kaon production threhsold can have sufficient energies for kaon production in a medium. This effect is similar to kaon production from multiparticles. As shown by Batko et al. [22], such processes increase the kaon yield by less than $10 \%$. Because these multiparticle effects are difficult to treat and have small contribution, they have been neglected in all previous studies on kaon production.

As the kaon production probability is much smaller than one in heavy ion collisions at subthreshold energies, it is usually treated by the perturbative method, i.e., the effect of kaon production on the dynamics of heavy ion collisions is neglected, first introduced in Ref. [13] and further extended in Ref. [14]. Specifically, a kaon is produced in a baryonbaryon or a meson-baryon interaction that is above the production threshold. The produced kaon then carries a probability given by the ratio of the kaon production cross section to the total baryon-baryon or meson-baryon cross section. Furthermore, to treat the rescattering of kaons and hyperons with other particles, one allows more than one pair of kaon and hyperon to be produced in such a collision. When these kaons and hyperons are scattered by other particles, their momenta are changed according to the differential cross section, which is usually taken to be isotropic, while changes of the momenta of the other particles are neglected. The final kaon yield and spectrum are then obtained by adding the probabilities of individual kaons and dividing by the number of kaons produced in each baryon-baryon or meson-baryon collision. We note that although many kaons are produced in the perturbative approach, each kaon actually correponds to a single kaon event as they are independent. The approximation introduced in this method is to assume that the medium through which the kaon propagates is the same for all these events and that the medium is not affected by kaon production and scattering. Compared with real kaon events, energy conservation is thus slightly violated in these theoretical events. But this should not have much effect on the kaon yield and spectrum as there is only one kaon in such an event and it scatters only a few times.

To study the chemical equilibration of kaons, we improve the model of Ref. [17] by including also kaon annihilation by hyperon, using the cross sections determined from the meson-baryon interactions via the detailed balance relations. Because of strangeness conservation, a kaon is produced together with a hyperon. As the kaon production probability is much less than one in heavy ion collisions at subthreshold energies, there is only one hyperon in an event in which a kaon is produced. Kaon annihilation can thus occur only when there is a collision between the same pair of kaon and hyperon that is produced in the baryon-baryon or mesonbaryon interaction. As shown in Ref. [11] via the kinetic

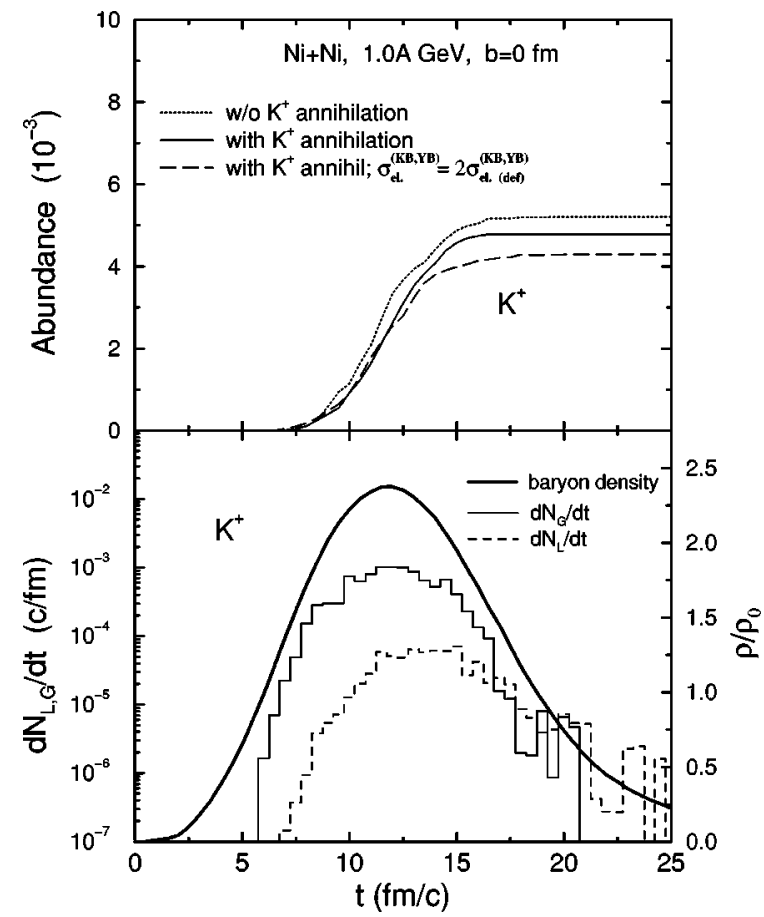

FIG. 1. Top panel: Time evolution of kaon abundance in $\mathrm{Ni}+\mathrm{Ni}$ collisions at $1 A \mathrm{GeV}$ and impact parameter $b=0$. Dotted curve is the result without kaon absorption while solid and dashed curves are results with kaon absorption from using default $K B$ and $Y B$ elastic scattering cross sections and twice the cross sections, respectively. Bottom panel: Time evolution of kaon production rate (solid curve) and absorption rate (dashed curve) as well as the central baryon density (thick solid curve).

equation, the annihilation between such a pair of particles that are produced simultaneously as a result of the $U(1)$ charge conservation would lead to an equilibration described by the canonical ensemble.

To illustrate the physics of kaon chemical equilibration in heavy ion collisions, we consider $\mathrm{Ni}+\mathrm{Ni}$ collisions at $1 A \mathrm{GeV}$ and impact parameter $b=0 \mathrm{fm}$, which is below the threshold for both kaon and antikaon production in nucleon-nucleon interactions. Similar results are obtained for collision energies at $2 \mathrm{~A} \mathrm{GeV}$, which is above the kaon production threshold but below the antikaon production threshold in nucleon-nucleon interactions. In the top panel of Fig. 1 , the time evolution of the kaon abundance for the scenarios with (solid curve) and without (dotted curve) kaon annihilation are given. It is seen that including kaon annihilation by hyperon reduces the final kaon yield by only about $10 \%$. As shown in the lower panel of Fig. 1, the kaon production rate (solid curve) is appreciable only when the nuclear density (thick solid curve) is high. The effect due to kaon annihilation is better illustrated by the kaon annihilation rate (dashed curve) shown in the bottom panel of Fig. 1. We see that the annihilation rate is negligible during the high density stage when most kaons are produced. This result thus justifies the neglect of kaon annihilation in previous studies. Although the kaon annihilation rate is small, it becomes comparable to the kaon production rate at about $17 \mathrm{fm} / c$, indicating that the kaon yield eventually approaches chemical equilibrium. 


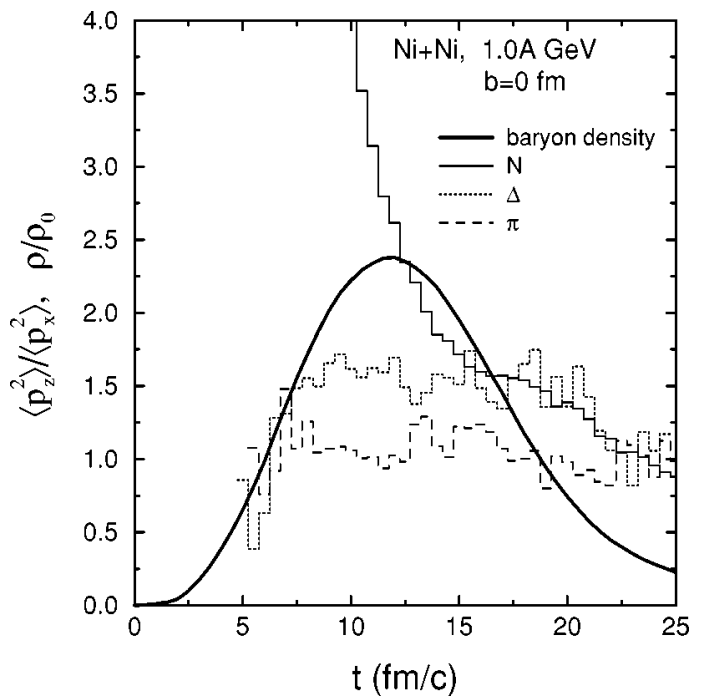

FIG. 2. Time evolution of the ratio $\left\langle p_{z}^{2}\right\rangle /\left\langle p_{x}^{2}\right\rangle$ for nucleons (solid curve), deltas (dotted curve), and pions (dashed curve). The central baryon density as a function of time is given by the thick solid curve.

We note that the baryon density at which kaons reach chemical equilibrium is about $1.2 \rho_{0}$, where $\rho_{0} \approx 0.16 \mathrm{fm}^{-3}$ is the normal nuclear matter density.

To better understand kaon production in heavy ion collisions, it is useful to know if the system is close to thermal equilibrium when kaons are produced. For this purpose, we have evaluated the average squared momentum of all particles both in the beam direction, $\left\langle p_{z}^{2}\right\rangle$, and in the transverse direction, $\left\langle p_{x}^{2}\right\rangle$. The ratio $\left\langle p_{z}^{2}\right\rangle /\left\langle p_{x}^{2}\right\rangle$ can then be used to characterize the degree of thermal equilibrium, with a value of one corresponding to complete thermal equilibrium. In Fig. 2, we show the time evolution of this ratio for nucleons (solid curve), deltas (dotted curve), and pions (dashed curve) in the central volume of $8 \mathrm{fm}^{3}$. It is seen that at the time when most kaons are produced, both pions and deltas are close to thermal equilibrium while nucleons are not. Since kaons are dominantly produced from pion and nucleon interactions (about 70\%), our results thus show that they are mostly produced from a dense but not thermally equilibrated hadronic matter.

According to the kinetic theory [11], the chemical equilibration time in the canonical formalism is given by $\tau_{0}^{C}$ $=V / L=N_{K} /\left(d N_{L} / d t\right)$, where $V$ is the volume of the region where kaon annihilation occurs, $L$ is the momentum averaged cross section for kaon annihilation, and $N_{K}$ and $d N_{L} / d t$ are the kaon number and its absorption rate, respectively. At time $t=12 \mathrm{fm} / c$ when the kaon absorption rate is largest, we have from Fig. $1, N_{K}=4.2 \times 10^{-3}$ and $d N_{L} / d t=7.0$ $\times 10^{-5}$, which give a kaon chemical equilibration time of $60 \mathrm{fm} / c$ if the system is prevented from expanding from $t$ $=12 \mathrm{fm} / c$. On the other hand, the thermal average of the kaon annihilation cross section $\sigma_{K Y \rightarrow \pi N}$ is about $0.25 \mathrm{fm}^{2}$ at temperature $T=75 \mathrm{MeV}$, and it changes by less than $20 \%$ for $50<T<100 \mathrm{MeV}$ due to the exothermic nature of the annihilation process. The above value for the kaon chemical equilibration time implies that the effective volume in which kaon annihilation occurs is about $15 \mathrm{fm}^{3}$. Since the chemical equilibration time for kaons is much longer than the heavy ion collision time, we would normally expect them not to reach chemical equilibrium during the collisions. However, since the kaon production rate decreases strongly as the temperature decreases due to the large threshold of the production process, its value can thus become comparable to the annihilation rate when the system expands and cools. When this happens for a later stage of heavy ion collisions as shown in the transport model results of Fig. 1, kaons can then reach chemical equilibrium. We thus note that, since most kaons are produced in the dense but nonequilibrium stage, the chemical equilibration time could be overestimated when determined in the normal way. Furthermore, the chemical freeze-out temperature extracted from the final kaon abundance in thermal model analysis does not provide sufficient information on the dynamics of kaon production.

The effect due to kaon annihilation depends on the magnitude of kaon and hyperon elastic scattering cross sections with other particles. If there are no such scatterings, e.g, if these cross sections are set to zero, then the produced kaon and hyperon would simply move away from each other without further interactions, leading to a result similar to that without kaon annihilation. On the other hand, larger kaon and hyperon scattering cross sections with other particles would force them into a smaller region, thus increasing the kaon annihilation rate. This is demonstrated in the top panel of Fig. 1 by the dashed curve, which is obtained by taking the kaon and hyperon scattering cross sections with other baryons to be twice the default values. It is seen that these larger cross sections indeed further reduce the kaon yield.

We also study antikaon production within the relativistic transport model, where antikaons are produced not only from baryon-baryon and meson-baryon interactions but also from meson-hyperon interactions. The cross sections for antikaon production from both baryon-baryon and meson-baryon interactions are taken from an analysis based on the mesonexchange model [23]. For antikaon production from the meson-hyperon interactions, the cross sections are obtained from the empirical cross sections for $K^{-}$absorption by nucleon to form a pion and a hyperon [24]. As first pointed out in Ref. [25], antikaon production in heavy ion collisions at subthreshold energies is mainly due to the meson-hyperon interactions. For antikaon annihilation, it is dominated by the reaction $\bar{K} N \rightarrow \pi Y$, and this has already been included in previous transport model studies [17,26,27]. We note that to account for the observed enhancement of antikaon production in heavy ion collisions at subthreshold energies, it has been shown in Refs. $[17,26,27]$ that a dropping of antikaon mass due to medium effects is needed.

The results for antikaon production in $\mathrm{Ni}+\mathrm{Ni}$ collisions at $1 A \mathrm{GeV}$ and impact parameter $b=0 \mathrm{fm}$ are shown in Fig. 3. In the top panel, the time evolution of antikaon abundance is shown for the cases with (solid curve for default kaon and hyperon elastic scattering cross sections and dashed curve for twice the default cross sections) and without (dotted curve) kaon annihilation. The reduction of antikaon yield when kaon annihilation is allowed is due to the reduction in the production probability of hyperons, which contribute most to 


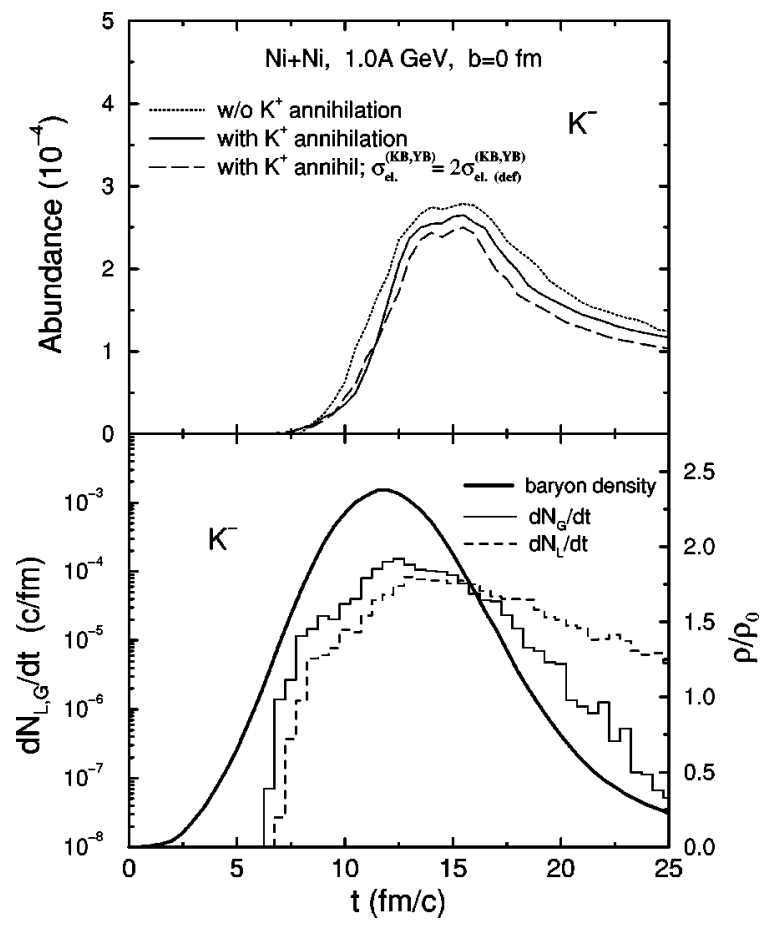

FIG. 3. Same as Fig. 1 for antikaons.

antikaon production. The time evolution of the antikaon production and annihilation rates are shown in the bottom panel of Fig. 3 by the solid and dashed curves, respectively. Similar to kaon production, we find that antikaons are mostly produced in the high density stage of heavy ion collisions.
Because of the large baryon density, their annihilation rate through the reaction $\bar{K} N \rightarrow \pi Y$ is larger than the kaon annihilation rate. As a result, antikaons approach chemical equilibrium even in the earlier stage of heavy ion collisions. However, they eventually fall out of equilibrium as shown in the lower panel of Fig. 3.

To summarize, we have improved the perturbative treatment of strange particle production in the relativistic transport model by including the annihilation of kaon and hyperon that are produced as a pair due to strangeness conservation. For heavy ion collisions at energies below the threshold for strange particle production in nucleon-nucleon interactions, we find that both kaons and antikaons are largely produced during the high density stage of the collisions when the system has not reached thermal equilibrium. Because of their large annihilation cross sections in dense nuclear matter, antikaons are near chemical equilibrium much earlier. For kaons, their abundance at the high density stage is far from equilibrium, and it only becomes close to the equilibrium value during the expansion stage of heavy ion collisions when the production rate is small and comparable to the annihilation rate. The small kaon annihilation rate in heavy ion collisions thus justifies the neglect of kaon annihilation in previous transport model studies of subthreshold kaon production.

This work was supported by the National Science Foundation under Grant No. PHY-9870038, the Welch Foundation under Grant No. A-1358, and the Texas Advanced Research Program under Grant No. FY99-010366-0081.
[1] P. Braun-Munzinger, I. Heppe, and J. Stachel, Phys. Lett. B 465, 15 (1999).

[2] J.S. Hamieh, K. Redlich, and A. Tounsi, Phys. Lett. B 486, 61 (2000).

[3] E.L. Bratkovskaya et al., Nucl. Phys. A675, 661 (2000).

[4] G.E. Brown, M. Rho, and C. Song, Nucl. Phys. A690, 184 (2001).

[5] FOPI Collaboration, J. Ritman, Z. Phys. A 352, 355 (1995).

[6] G.Q. Li, C.M. Ko, and B.A. Li, Phys. Rev. Lett. 74, 235 (1995); G.Q. Li and C.M. Ko, Nucl. Phys. A594, 460 (1995).

[7] P. Chung et al., Phys. Rev. Lett. 85, 940 (2000).

[8] S. Pal, C.M. Ko, Z.W. Lin, and B. Zhang, Phys. Rev. C 62, 061903(R) (2000).

[9] R. Barth et al., Phys. Rev. Lett. 78, 4007 (1997); C. Sturm et al., ibid. 88, 39 (2001).

[10] C. Fuchs et al., Phys. Rev. Lett. 86, 1974 (2001).

[11] C. M. Ko, V. Koch, Z. W. Lin, K. Redlich, M. Stephanov, and X. N. Wang, Phys. Rev. Lett. 86, 5438 (2001).

[12] C.M. Ko and G.Q. Li, J. Phys. G 22, 1673 (1996); W. Cassing and E.L. Bratkovskaya, Phys. Rep. 308, 65 (1999); S. Bass et al., Prog. Part. Nucl. Phys. 42, 313 (1999).

[13] J. Randrup and C.M. Ko, Nucl. Phys. A343, 519 (1980).

[14] X.S. Fang, C.M. Ko, and Y.M. Zheng, Nucl. Phys. A556, 499 (1993).

[15] J. Aichelin and C.M. Ko, Phys. Rev. Lett. 55, 2661 (1985);
X.S. Fang, C.M. Ko, G.Q. Li, and Y.M. Zheng, Phys. Rev. C 49, 1139 (1994); Nucl. Phys. A575, 766 (1994); G.Q. Li and C.M. Ko, Phys. Lett. B 349, 405 (1995).

[16] S.W. Huang et al., Phys. Lett. B 298, 41 (1993); T. Maruyama et al., Nucl. Phys. A573, 653 (1994); C. Hartnack et al., ibid. A580, 643 (1994).

[17] G.Q. Li, C.H. Lee, and G.E. Brown, Nucl. Phys. A625, 372 (1997).

[18] C.M. Ko, Q. Li, and R. Wang, Phys. Rev. Lett. 59, 1084 (1987); C.M. Ko and Q. Li, Phys. Rev. C 37, 2270 (1988); Q. Li, J.Q. Wu, and C.M. Ko, ibid. 39, 849 (1989); C.M. Ko, Nucl. Phys. A495, 321c (1989).

[19] G.Q. Li and C.M. Ko, Nucl. Phys. A594, 439 (1995).

[20] K. Tsushima, S.W. Huang, and A. Faessler, Phys. Lett. B 337, 245 (1994); J. Phys. G 21, 33 (1995).

[21] D.B. Kaplan and A.E. Nelson, Phys. Lett. 175B, 57 (1986).

[22] G. Batko, J. Randrup, and T. Vetter, Nucl. Phys. A536, 786 (1992).

[23] A. Sibirtsev, W. Cassing, and C.M. Ko, Z. Phys. A 358, 101 (1997).

[24] J. Cugnon, P. Deneye, and J. Vandermeulen, Phys. Rev. C 41, 1701 (1990).

[25] C.M. Ko, Phys. Lett. 120B, 294 (1983); 138B, 361 (1984).

[26] G.Q. Li, C.M. Ko, and X.S. Fang, Phys. Lett. B 329, 149 (1994).

[27] W. Cassing et al., Nucl. Phys. A614, 415 (1997). 